\title{
Solving Multi-objective Aeroassisted Spacecraft Trajectory Optimization Problems Using Extended NSGA-II
}

\author{
Runqi Chai, Al Savvaris ${ }^{\dagger}$ and Antonios Tsourdos $\ddagger$ \\ Cranfield University, Bedfordshire, MK43 0AL, United Kingdom \\ Senchun Chai ${ }^{\S}$ \\ Beijing Institute of Technology, Beijing, 100081, China
}

\begin{abstract}
This paper focuses on the application of an extended NSGA-II algorithm to the aeroassisted spacecraft trajectory optimization problems. A new multi-objective spacecraft optimal control model is formulated and parameterized using discretization method. The resulting multi-objective nonlinear programming problem is then solved via the multiobjective evolutionary solver. In order to deal with path constraints that naturally arise in practical trajectory planning problems, the original NSGA-II approach is extended by introducing a new constraint handling strategy. Simulation results are provided to illustrate the effectiveness and feasibility of the enhanced NSGA-II algorithm in dealing with spacecraft trajectory optimization problems.
\end{abstract}

\section{Introduction}

Spacecraft trajectory optimization problems have attracted significant attentions over the past couple of decades. $^{1,2}$ This type of problem is usually solved by applying direct transcription algorithms such as direct collocation, ${ }^{3}$ direct multiple shooting ${ }^{4,5}$ or pseudospectral methods. ${ }^{6,7}$ All the direct methods aim to transcribe the continuous-time trajectory optimization (optimal control) problems to a Nonlinear Programming problem (NLP). ${ }^{3,8}$ The resulting NLP can then be solved numerically by well-developed algorithms such as gradient-based methods or evolutionary-based algorithms.

In recent years, evolutionary-based methods have become more popular in the application of optimal control problems. ${ }^{8}$ The main difference between traditional gradient methods and derivative-free (evolutionarybased) methods is that derivative-free methods such as Genetic Algorithm (GA), ${ }^{9}$ Artificial Bee Colony $(\mathrm{ABC})$ and Particle Swam Optimization (PSO), 8,10 do not need the calculation of gradient information. Moreover, evolutionary algorithms requires no initial guess since the initial population is chosen randomly, and it is more likely than gradient methods to locate the global minimum. Contributions made to apply these approaches can be found in literatures. For example, Yokoyama et al. ${ }^{11}$ implemented GA to solve a constrained space plane reentry problem. Kamesh et al. ${ }^{12}$ applied a Hybrid Genetic Algorithm Collocation to analyze an Earth-Mars orbit transfer problem. Although most of these applications are targeted at singleobjective problems, in reality, for space vehicle trajectory design, most missions may contain multiple mission requirements and this brings the development of multiple objective evolutionary trajectory optimization. ${ }^{1,13}$

The mission scenario investigated in this paper focuses on the atmospheric skip hopping, targeting the entry into the atmosphere down to a predetermined altitude point and the required controls involved in returning back to Low Earth Orbit (LEO). Studies can be found in the literature regarding the skip reentry of deep-space spacecraft with high speed over first cosmic velocity. However in the scenario considering in this paper, a high thrust engine would be necessary for the SMV to return to low earth orbit. An example

*Ph.D. Student, School of Aerospace Transport and Manufacturing, r.chai@cranfield.ac.uk, Student Member AIAA.

$\dagger$ Reader, School of Aerospace Transport and Manufacturing, a.savvaris@cranfield.ac.uk, Senior Member AIAA

¥Professor, School of Aerospace Transport and Manufacturing, a.tsourdos@cranfield.ac.uk, Senior Member AIAA

$\S$ Associate Professor, School of Automation, chaisc97@163.com. 
of a single-hop mission can be seen in Fig.1. Please note that for some other missions they might involve multiple hops and not just one. The general skip-entry can be divided into five phases: initial roll, down

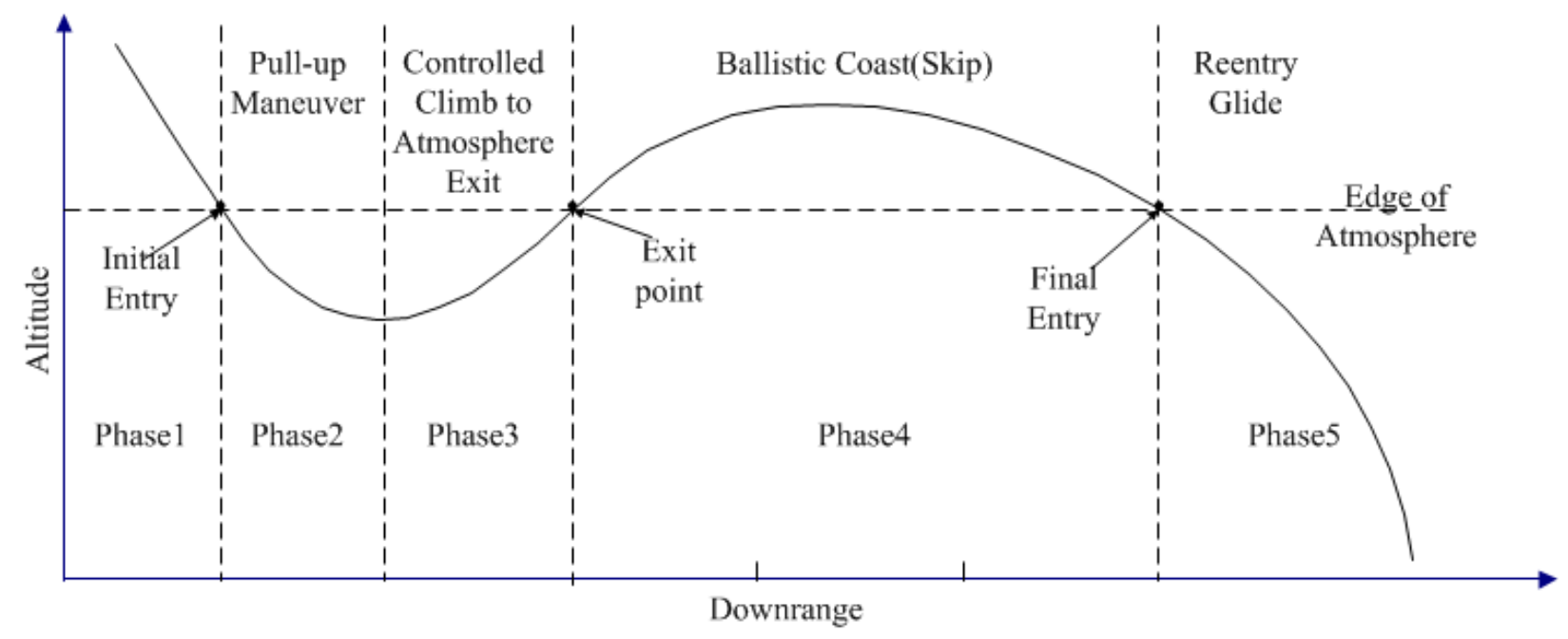

Figure 1. Mission profile

control, up control, Kepler and final entry. Considering the mission of the SMV is to overfly the ground target at specific altitude, the most challenging two phases (i.e. phase 2 and 3 ) will be considered in this paper.

To solve the constrained spacecraft trajectory optimization problems, an extended multi-objective evolutionary algorithm is constructed and applied. The fundamental framework of this algorithm is based on the Nondominated Sorted Genetic Algorithm-II (NSGA-II). ${ }^{14}$ The original algorithm is modified by embedding a new constraint handling strategy so as to deal with the flight path constraints. This will be further discussed in Section III.

\section{Multi-Objective Aeroassisted Spacecraft Trajectory Optimization Problem}

\section{A. Dynamic model}

Consider the motion of an aeroassisted vehicle modeled as a point mass over a spherical non-rotating Earth. The vehicle re-enters the atmosphere at a predetermined altitude for observation and gathering of information of inaccessible areas. Once this altitude point is reached, the spacecraft fires its engine and starts the ascent phase, exiting the atmosphere and returning back to LEO. During the flight, the differential equations of motion are given as: ${ }^{1,2}$

$$
\begin{aligned}
& \dot{r}=V \sin \gamma \\
& \dot{\theta}=\frac{V \cos \gamma \sin \psi}{r \cos \phi} \\
& \dot{\phi}=\frac{V \cos \gamma \cos \psi}{r} \\
& \dot{V}=\frac{T \cos \alpha-D}{m}-g \sin \gamma \\
& \dot{\gamma}=\frac{L \cos \sigma+T \sin \alpha}{m V}+\left(\frac{V^{2}-g r}{r V}\right) \cos \gamma \\
& \dot{\psi}=\frac{L \sin \sigma}{m V \cos \gamma}+\frac{V}{r} \cos \gamma \sin \psi \tan \phi \\
& \dot{m}=-\frac{T}{I_{s p} g}
\end{aligned}
$$

where $x=[r, \theta, \phi, V, \gamma, \psi, m]^{T}$ are state variables representing: radial distance, longitude, latitude, speed, flight path angle, heading angle and mass, respectively. $u=[\alpha, \sigma, T]^{T}$ are control variables of angle of attack, bank angle and thrust. For simplicity reasons, the dynamic model described in Eq.(1) is abbreviated as $\dot{x}=f(x, u), x(0)=x_{0}$, where $x \in \Re^{7}$ and $u \in \Re^{3} . x_{0}=\left[r_{0}, \theta_{0}, \phi_{0}, V_{0}, \gamma_{0}, \psi_{0}, m_{0}\right]$ are the initial conditions for the state variables. 
The atmosphere and aerodynamic model can be summarised as:

$$
\begin{array}{ll}
g=\frac{\mu}{r^{2}} & \rho=\rho_{0} \exp \frac{r-r_{e 0}}{h_{s}} \\
L=\frac{1}{2} \rho V^{2} C_{L} S & D=\frac{1}{2} \rho V^{2} C_{D} S \\
C_{D}=C_{D 0}+C_{D 1} \alpha+C_{D 2} \alpha^{2} & C_{L}=C_{L 0}+C_{L 1} \alpha
\end{array}
$$

where $S=249.91 \mathrm{~m}^{2}$ is reference area, $\rho$ is the density of the atmosphere and $\rho_{0}=1.2250 \mathrm{~kg} / \mathrm{m}^{3}$ is the density of the atmosphere at sea-level. $r_{e 0}=6378.135 \mathrm{~km}$ is earth radius, $L$ and $D$ are the lift and drag whereas $C_{L}$ and $C_{D}$ are the corresponding lift and drag coefficients. $g$ is the gravitational acceleration.

A detailed description in terms of the entry reference frames and aerodynamic forces can be found in Fig.2.

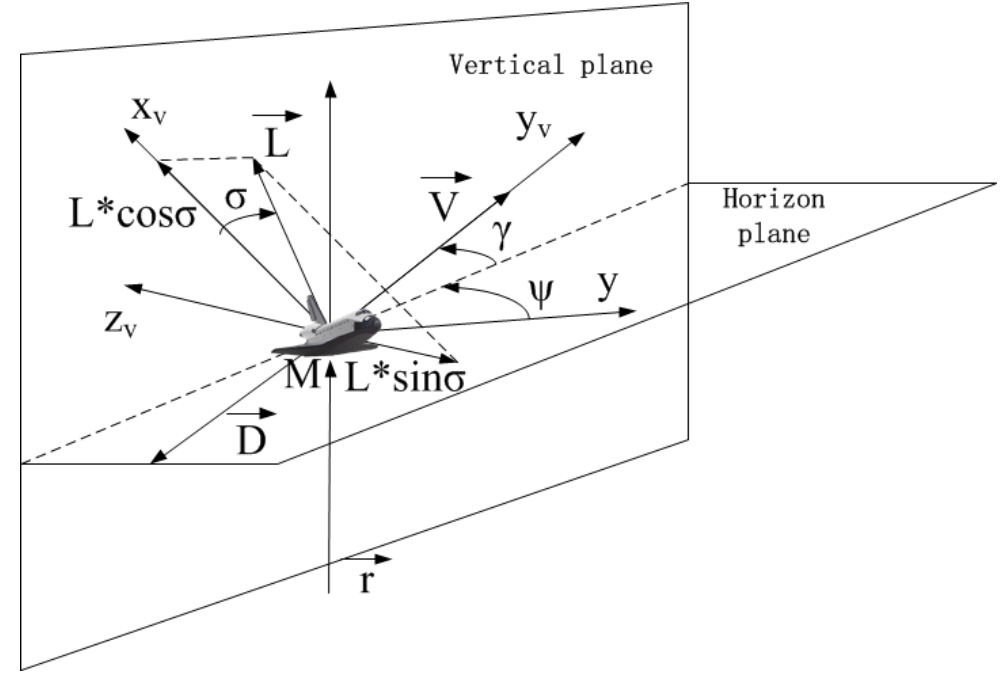

Figure 2. Aerodynamic forces

During the mission, each state and control variable should satisfy strict box constraints, which can be described as $x_{\min } \leq x \leq x_{\max }$ and $u_{\min } \leq u \leq u_{\max }$. In addition to taking the path constraints into account, in this investigation the heating rate $Q$, dynamic pressure $P_{d}$ and load factor $n_{l}$ are also considered. The path constraints can be formulated as:

$$
\begin{gathered}
\dot{Q}_{d}=K_{Q} \rho^{0.5} V^{3.07} q_{a}<\dot{Q}_{d \max } \\
P_{d}=\frac{1}{2} \rho V^{2}<P_{d \max } \\
n_{L}=\frac{\sqrt{L^{2}+D^{2}}}{m g}<n_{\max }
\end{gathered}
$$

where $q_{a}=\left(c_{0}+c_{1} \alpha+c_{2} \alpha^{2}+c_{3} \alpha^{3}\right)$.

\section{B. Mission objectives}

In the past, early studies on spacecraft trajectory optimization problems usually focused on single objective. However, in order to take more practical requirements into account, this type of problem should be constructed containing multiple objectives and this is where nowadays the majority of research is focusing on. To take more of the mission requirements into account, four objectives are considered. The first objective is to minimize the final time so as to complete a reconnaissance mission in the shortest possible time interval. In addition, as mentioned in, ${ }^{1}$ minimizing the total aerodynamic heating is also chosen as one of the objectives since the vehicle structure integrity is largely affected by the aerodynamic heating. While the third objective is to maximize the final velocity so that the vehicle can have higher kinetic energy, to enable the vehicle to have more flexibility to manoeuvre back into orbit. Moreover, in this mission scenario to ensure the aeroassisted vehicle has enough fuel to carry-out several skip hops, the final objective is set to minimize the fuel consumption, i.e., maximize the final mass value, during the whole manoeuvre. Therefore, the objective functions selected for the analysis are: 
1). Minimizing the final time:

$$
\min J_{1}=t_{f}
$$

2). Minimizing the total aerodynamic heating:

$$
\min J_{2}=\int_{t_{0}}^{t_{f}} \dot{Q}(t) d t
$$

3). Maximizing the final velocity:

$$
\max J_{3}=V\left(t_{f}\right)
$$

4). Maximizing the final mass:

$$
\max J_{4}=m\left(t_{f}\right)
$$

\section{Extended NSGA-II Algorithm}

\section{A. Discrete model}

To solve the continuous trajectory optimization problem, discrete algorithms should be implemented such that the continuous optimal control problem can be converted to static Nonlinear Programming (NLP) problem. The control variables can be approximated by interpolation at $N_{k}$ th discretized time nodes $\left[t_{1}, t_{2}, \ldots, t_{N_{k}}\right]$. Then the equations of motion are integrated with a fourth order Runge-Kutta method. The discretized formulation of problem (1) is then given by:

$$
\begin{array}{cl}
\operatorname{minimize} & J=\left[J_{1}, J_{2}, J_{3}, J_{4}\right] \\
\text { subject to } & x_{k+1}=x_{k}+h_{k} \sum_{i=1}^{s} b_{i} f\left(x_{k i}, u_{k i}\right) \\
& x_{k i}=x_{k}+h_{k} \sum_{j=1}^{s} a_{i j} f\left(x_{k j}, u_{k j}\right) \\
& g\left(x_{k i}, u_{k i}\right) \geq 0 \\
& x(0)=x_{0} \\
& i, j=1, \ldots, s, \quad k=0, \ldots, N_{k}-1
\end{array}
$$

where $N_{k}$ is the number of discretized time nodes, while $g$ stands for the inequality constraints described in Eq.(3). Following the use of the discretization technique, the resulting multi-discipline NLP is solved by an extended NSGA-II algorithm.

\section{B. Extended NSGA-II algorithm}

The multi-objective stochastic solver used for this work is the extended NSGA-II algorithm. This algorithm utilises the mechanism of crossover and mutation operator to generate offspring population, and employs fast non-dominated sorting approach to determine the non-dominant rank for each candidate solution. During the optimization procedure, the first front is generated as the set of solutions that has the highest fitness value and is not dominated by any other solutions in the current population. For completeness, a brief description of this extended NSGA-II optimization algorithm is introduced.

There are four main procedures for the proposed algorithm, initialization, selection (based on the nondominated sort), crossover and mutation. In the initialization part, according to the discrete-time model given by Eq.(8), the optimization parameters should be initialized at all the discrete time nodes. This can be written as:

$$
\begin{gathered}
\alpha=\alpha_{\text {min }}+\operatorname{rand}(\cdot) \times\left(\alpha_{\text {max }}-\alpha_{\text {min }}\right) \\
\sigma=\sigma_{\text {min }}+\operatorname{rand}(\cdot) \times\left(\sigma_{\text {max }}-\sigma_{\text {min }}\right) \\
T=T_{\text {min }}+\operatorname{rand}(\cdot) \times\left(T_{\text {max }}-T_{\text {min }}\right)
\end{gathered}
$$

Following the population initialization, the mutation and crossover strategy used in this paper can be found in. ${ }^{14}$ The classic NSGA-II determines the dominance relationship between individuals only by comparing the objective function values. When solving the trajectory optimization problem with constraints, NSGA-II lacks the rule to handle the infeasible candidates. This paper extends the original NSGA-II algorithm by using the violation degree of constraints $V$ and this will be the primary metric to define the V-based dominant relationship. ${ }^{15}$ 
The constraint violation for each individual among the current population can be calculated as:

$$
V_{i}=\sum_{j=1}^{I} \mu_{g_{j}}(u)+\sum_{k=1}^{E} \mu_{h_{k}}(u), \quad i=1,2 \ldots N P
$$

where $E$ and $I$ are the total number of equality and inequality constraints, respectively. $N P$ is the size of population. $\mu_{g_{j}}$ and $\mu_{h_{k}}(u)$ are constraint violation functions corresponding to the inequality and equality constraints, respectively. The definition of these function can be found in.,15 Based on the $V$ function established above, each individual can be associated with all the constraints. In this way, the value of the $V$ function can directly reflect the magnitude of the solution infeasibility.

\section{V-based dominant sorting}

The violation degree of the $i$ th individual is the sum violation of all the constraints and based on this definition, the extended V-based dominant rule " $\succ$ " is defined as follows:

Definition 1 (V-based dominant rule " $\succ$ ") For two individuals $u_{1}$ and $u_{2}$ in the current population, $u_{2}$ is said to be dominated by $u_{1}$ if and only if one of the following relationships is satisfied:

1. $V(u(2))>V(u(1))>0$.

2. $V(u(1))=0, V(u(2))>0$.

3. $V(u(1))=V(u(2))=0$, and for each objective function $i, J_{i}(u(1))<J_{i}(u(2))$ is satisfied (Classic dominance definition).

As shown in Definition.1, the feasible individual always dominates the infeasible one, while the individual with smaller violation degree always dominates the one with higher violation degree. After the dominance relationships are determined, the proposed algorithm applies a fast non-dominated sorting approach to divide the population into different ranks. ${ }^{14}$ It should be noted that for constrained spacecraft trajectory optimization problems, it is likely that all of the individual among the population are infeasible solution in the first several generations. Then, three problem types can be introduced. If all the individuals in the current population are infeasible solutions, the problem type is set to 0 , whereas if some of the individuals are feasible solutions, the problem type is set to 0.5. Correspondingly, Problem type $=1$ means all the candidates among the population are feasible solutions. To increase the algorithm efficiency, for the first several generations (Problem type $=0$ ), the non-dominant rank can be simply assigned by sorting the violation degree of the individuals. The overall structure of this enhanced NSGA-II optimization algorithm can be summarised as follows:

1. Initialize the population $\left(u_{1}, \ldots, u_{N_{p}}\right)$ and other control parameters of the proposed algorithm.

2. For each candidate, calculate the objective function values $J$ and the violation degree according to Eq.(10).

3. Generate offspring population $Q_{t}$ by using the recombination and mutation processes, ${ }^{14}$ and combine it with the parent population $P_{t}$ to obtain $R_{t}\left(R_{t}=Q_{t} \cup P_{t}\right)$.

4. Specify the problem type by checking the violation degree of individuals.

5. If Problem type $=0$, then get all non-dominated ranks by sorting the violation degree (e.g. assign the candidate trajectory having the smallest violation degree in the first front $F_{1}$ ).

6. If Problem type $=0.5$ or 1 , then assign all non-dominated ranks using the V-based dominant rule.

7. According to the selection operator based on the crowding distance, ${ }^{14}$ select the best $N_{p}$ individuals as the candidates of the new generation $S_{t}$.

8. Repeat step 2-7 until the maximum iteration number is achieved. 
Table 1. Control parameters for NSGA-II algorithm

\begin{tabular}{lr}
\hline \hline Item & Value \\
\hline Population size & 250 \\
Maximum number of generations & 10000 \\
Tournament selection scale & 3 \\
Probability of crossover & 0.8 \\
Probability of mutation & 0.2 \\
\hline \hline
\end{tabular}

\section{Simulation results}

For the extended NSGA-II simulation, all of the control parameters are tabulated in Table 1.

The box constraint values (lower and upper bounds for each variable) are given in Table 2. The path constraints maximum allowable values for heating, dynamic pressure and load factor are set as follows: $Q_{\max }=200 B T U ; P_{d \max }=13406.4583 P a ; n_{l \max }=2.5$.

Table 2. Initial conditions and box constraints for each variable

\begin{tabular}{c|c|c|c|c|c}
\hline \hline & $\mathrm{r}(\mathrm{m})$ & $\theta(\mathrm{deg})$ & $\phi(\mathrm{deg})$ & $\mathrm{V}(\mathrm{m} / \mathrm{s})$ & $\gamma(\mathrm{deg})$ \\
\hline Initial conditions & 6450451.9 & 0 & 0 & 7802.9 & -1 \\
Minimum & 6421191.1 & -180 & -70 & 609.6 & -80 \\
Maximum & 6450451.9 & 180 & 70 & 9144.0 & 80 \\
\hline \hline & $\psi(\mathrm{deg})$ & $\mathrm{m}(\mathrm{kg})$ & $\alpha(\mathrm{deg})$ & $\sigma(\mathrm{deg})$ & $T(N)$ \\
\hline Initial conditions & 90 & 92078.8 & 17.43 & -75 & 0 \\
Minimum & -180 & 20000.0 & 0 & -90 & 0 \\
Maximum & 180 & 92078.8 & 40 & 1 & $2 \times 10^{6}$ \\
\hline \hline
\end{tabular}

\section{A. Pareto-front solutions}

Pareto front solutions are plotted in Figs.3-5. The pareto fronts are projected onto three planes: Minimizing terminal time versus minimizing aerodynamic heating, maximizing terminal speed versus maximizing terminal mass, and minimizing aerodynamic heating versus maximizing terminal speed.

According to the mission objectives established in Eq.(4)-(7), it is observed that the objectives in Eq.(6) and Eq.(7) are contradicting each other. More precisely, maximizing the final velocity can only be achieved at the expense of fuel consumption (see Fig.4). This can also be reflected by the velocity and mass dynamics. In addition, it should be noted that one of the parameters, that can increase aerodynamic heating, is dynamic pressure. Dynamic pressure is functions of density and velocity. Since density in skip entry phase is relatively small compared to velocity, maximizing velocity in turn increases aerodynamic heating (see Fig.5). This leads to that the objective functions in Eq.(5) and Eq.(6) are also contradicting.

On the other hand, as can be seen from the aerodynamic heating equation, the total amount of heating is largely affected by the upper limit of integration $\left(t_{f}\right)$. Therefore, minimizing the terminal time (Eq.(4)) and minimizing the total aerodynamic heating (Eq.(5)) are highly correlated objectives (see Fig.3).

\section{B. Path constraint solutions}

To further analyze the performance of the enhanced NSGA-II algorithm, the path constraint results are tabulated in Table.3.

As can be seen from Table.3, by applying the proposed V-based dominant sorting strategy, all the candidate solutions in the obtained pareto-optimal set can satisfy the three path constraints. Therefore, the structural and thermal safety of the aeroassisted vehicle is guaranteed, which is the prerequisite for the validity of a constraint handling strategy for spacecraft trajectory optimization problems. 


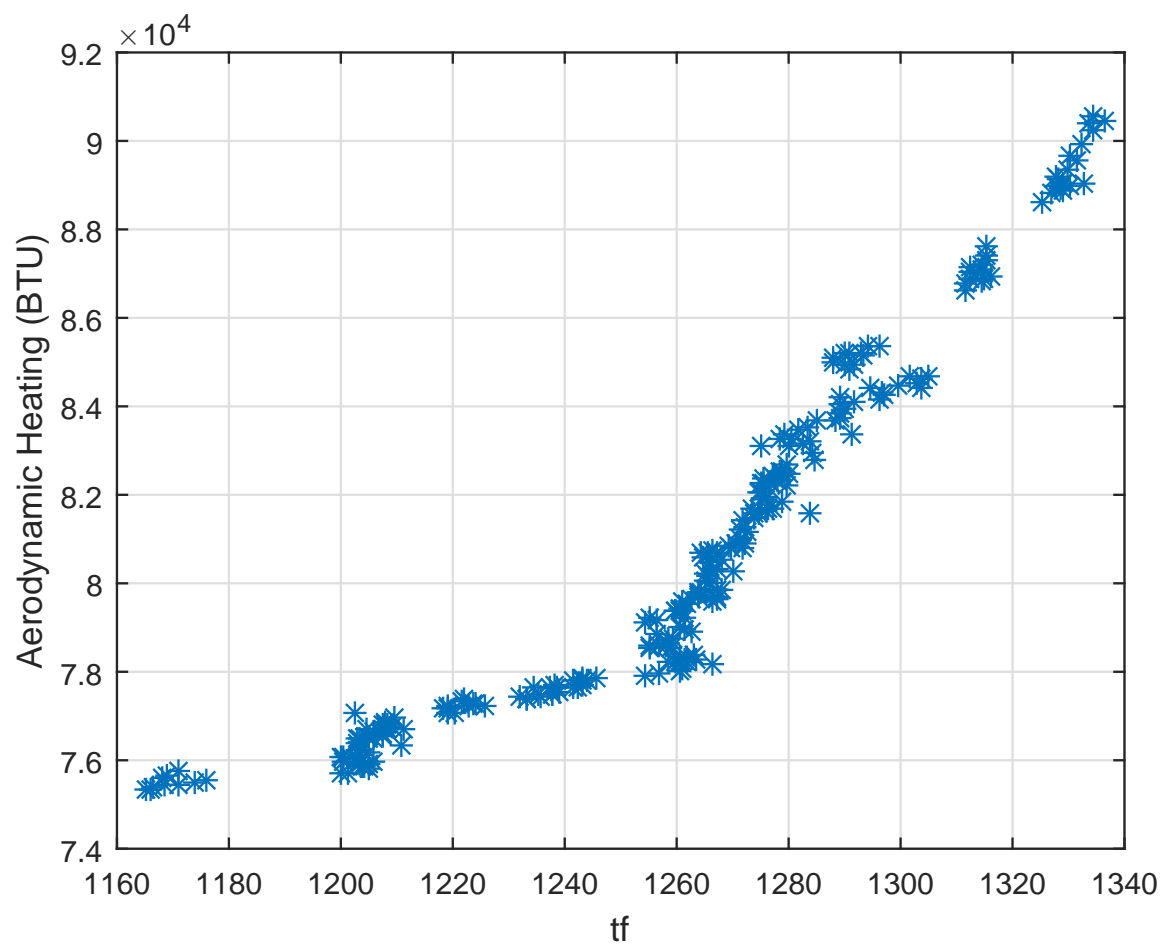

Figure 3. Pareto front obtained via extended NSGA-II



Figure 4. Pareto front obtained via extended NSGA-II 


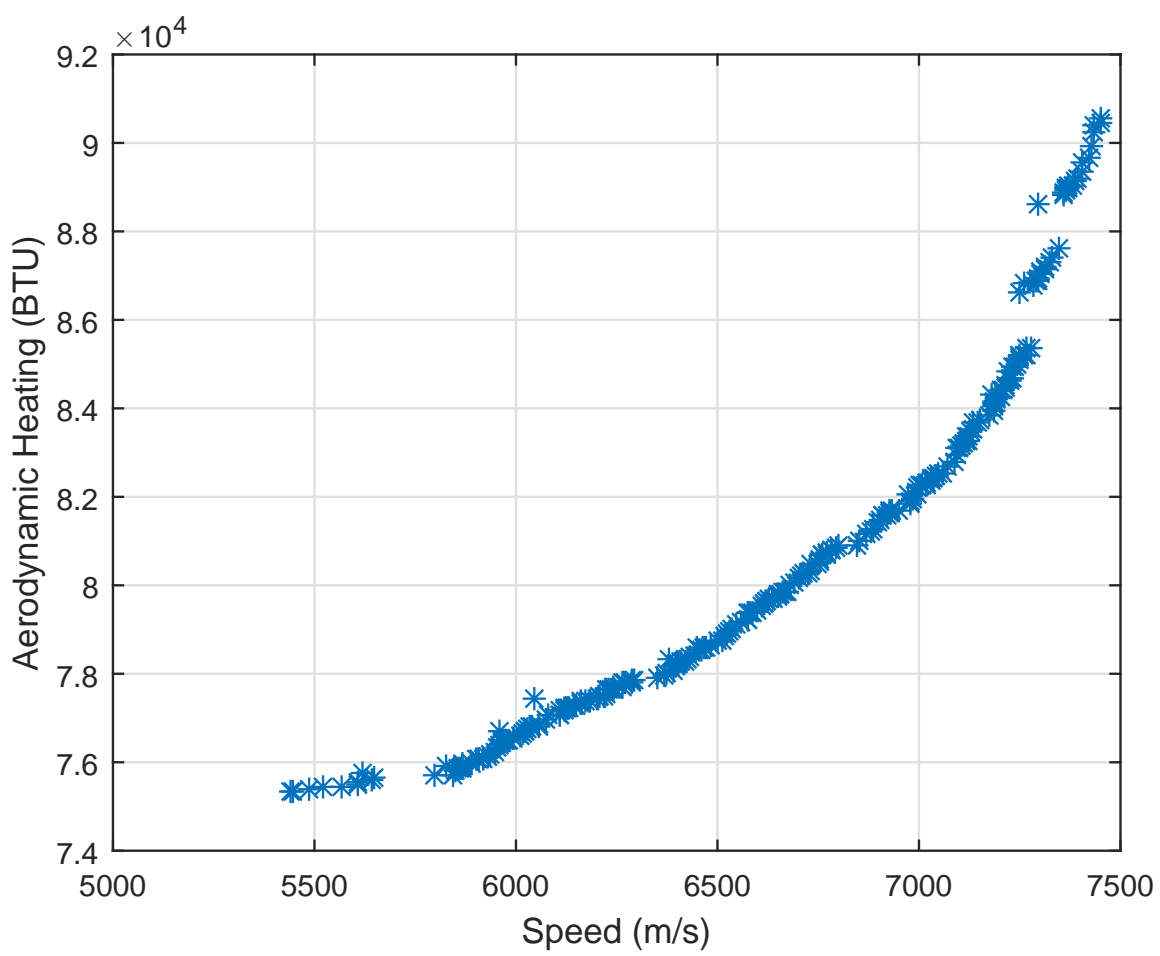

Figure 5. Pareto front obtained via extended NSGA-II

Table 3. Path constraint results

\begin{tabular}{c|c|c|c}
\hline \hline Extended NSGA-II & $Q_{\max }$ & $P_{\text {dmax }}$ & $n_{\operatorname{lmax}}$ \\
\hline \hline Maximum & 197.62 & 13202.04 & 2.37 \\
Violation degree & 0 & 0 & 0 \\
\hline
\end{tabular}

\section{Conclusions}

In this paper, an enhanced multi-objective evolutionary algorithm was constructed and applied to solve the aeroassisted spacecraft trajectory optimization problem. To deal with the path constraints, a new constraint handling strategy is embedded in the original NSGA-II framework. Simulation results show the trade-off and conflicting relationships between different mission objective. Moreover, all the obtained solutions confirm the effectiveness and feasibility of the extended evolutionary solver for solving spacecraft trajectory optimization problems. Therefore, the proposed solver can offer an efficient alternative for optimizing this type of problem.

\section{References}

\footnotetext{
${ }^{1}$ Chai, R., Savvaris, A., and Tsourdos, A., "Fuzzy physical programming for Space Manoeuvre Vehicles trajectory optimization based on hp-adaptive pseudospectral method," Acta Astronautica, Vol. 123, 2016, pp. 62-70, doi:http://dx.doi.org/10.1016/j.actaastro.2016.02.020.

${ }^{2}$ Senses, B. and Rao, A. V., "Optimal Finite-Thrust Small Spacecraft Aeroassisted Orbital Transfer," Journal of Guidance, Control, and Dynamics, Vol. 36, No. 6, 2013, pp. 1802-1810, doi:10.2514/1.58977.

${ }^{3}$ Betts, J. T., Practical Methods for Optimal Control and Estimation Using Nonlinear Programming, Cambridge University Press, 2009.

${ }^{4}$ Laurent-Varin, J., Bonnans, F., Berend, N., Haddou, M., and Talbot, C., "Interior-Point Approach to Trajectory Optimization," Journal of Guidance, Control, and Dynamics, Vol. 30, No. 5, 2007, pp. 1228-1238, doi:10.2514/1.18196.
} 
${ }^{5} \mathrm{Lu}$, P., "Entry Guidance Using Time-Scale Separation in Gliding Dynamics," Journal of Spacecraft and Rockets, Vol. 52 , No. 4, 2015, pp. 1253-1258, doi:10.2514/1.A33295.

${ }^{6}$ Benson, D. A., Huntington, G. T., Thorvaldsen, T. P., and Rao, A. V., "Direct Trajectory Optimization and Costate Estimation via an Orthogonal Collocation Method," Journal of Guidance, Control, and Dynamics, Vol. 29, No. 6, 2006, pp. $1435-1440$, doi: $10.2514 / 1.20478$.

${ }^{7}$ Jorris, T. R. and Cobb, R. G., "Multiple Method 2-D Trajectory Optimization Satisfying Waypoints and No-Fly Zone Constraints," Journal of Guidance, Control, and Dynamics, Vol. 31, No. 3, 2008, pp. 543-553, doi:10.2514/1.32354.

${ }^{8}$ Conway, B. A., "A Survey of Methods Available for the Numerical Optimization of Continuous Dynamic Systems," Journal of Optimization Theory and Applications, Vol. 152, No. 2, 2012, pp. 271-306, doi:10.1007/s10957-011-9918-z.

${ }^{9}$ Wall, B. J. and Conway, B. A., "Genetic algorithms applied to the solution of hybrid optimal control problems in astrodynamics," Journal of Global Optimization, Vol. 44, No. 4, 2008, pp. 493-508, doi:10.1007/s10898-008-9352-4.

${ }^{10}$ Pontani, M. and Conway, B. A., "Particle Swarm Optimization Applied to Space Trajectories," Journal of Guidance, Control, and Dynamics, Vol. 33, No. 5, 2010, pp. 1429-1441, doi: $10.2514 / 1.48475$.

${ }^{11}$ Yokoyama, N. and Suzuki, S., "Modified Genetic Algorithm for Constrained Trajectory Optimization," Journal of Guidance, Control, and Dynamics, Vol. 28, No. 1, 2005, pp. 139-144, doi:10.2514/1.3042.

${ }^{12}$ Subbarao, K. and Shippey, B. M., "Hybrid Genetic Algorithm Collocation Method for Trajectory Optimization," Journal of Guidance, Control, and Dynamics, Vol. 32, No. 4, 2009, pp. 1396-1403, doi:10.2514/1.41449.

${ }^{13}$ Tsourdos, A., Hughes, E. J., and White, B. A., "Fuzzy multi-objective design for a lateral missile autopilot," Control Engineering Practice, Vol. 14, No. 5, 2006, pp. 547-561, doi:http://dx.doi.org/10.1016/j.conengprac.2005.01.009.

${ }^{14}$ Deb, K., Pratap, A., Agarwal, S., and Meyarivan, T., "A fast and elitist multiobjective genetic algorithm: NSGA-II," IEEE Transactions on Evolutionary Computation, Vol. 6, No. 2, 2002, pp. 182-197, doi:10.1109/4235.996017.

${ }^{15}$ Chai, R., Savvaris, A., Tsourdos, A., and Chai, S., "Multi-objective trajectory optimization of Space Manoeuvre Vehicle using adaptive differential evolution and modified game theory," Acta Astronautica, Vol. 136, 2017, pp. 273-280, doi:http://dx.doi.org/10.1016/j.actaastro.2017.02.023. 
2017-09-14

\section{Solving multi-objective aeroassisted}

spacecraft trajectory optimization

problems using extended NSGA-II

\section{Chai, Runqi}

American Institute of Aeronautics and Astronautics

Chai R, Savvaris A, Tsourdos A, Chai S, Solving multi-objective aeroassisted spacecraft trajectory optimization problems using extended NSGA-II, Proceedings of AIAA SPACE and Astronautics Forum and Exposition 2017, 12-14 September 2017, Orlando, FL, USA, Paper number AIAA 2017-5193, pp. 1-9

http://dx.doi.org/10.2514/6.2017-5193

Downloaded from Cranfield Library Services E-Repository 